\title{
«Prozessintelligenz»: Gegenstand und Ziele der Studie
}

Elke Brucker-Kley, Denisa Kykalová und Thomas Keller

1.1 Forschungsgegenstand und Ausgangslage - 4

1.2 Studiendesign und Ergebnisse - 7

1.3 Das Rahmenwerk: Prozessintelligenz = Erfolgsintelligenz - 9

Literatur - 13 
«Intelligente Prozesse, «intelligentes Prozessmanagement», «iBPM» sind Schlagworte, die in erster Linie eingesetzt werden, um Technologien zu vermarkten. Die Begriffe lassen viel Raum für Interpretation und Assoziationen. Können Unternehmen den Hype ignorieren oder bietet «Prozessintelligenz» die Chance, das Prozessmanagement aus einem anderen Blickwinkel zu betrachten und weiterzuentwickeln? Doch was ist Prozessintelligenz? Welche Lösungsansätze, Erfahrungen und Erfolgsmuster gibt es bereits in Unternehmen? Welche Methoden und Werkzeuge kommen zum Einsatz, um Prozesse «intelligenter» zu machen? Diesen Fragen hat sich die Business-Process-Management-Studie 2015 gestellt, mit der das Institut für Wirtschaftsinformatik der Zürcher Hochschule für Angewandte Wissenschaften School of Management and Law seit 2011 regelmässig Status quo und Best Practices im deutschsprachigen Raum erhebt.

\subsection{Forschungsgegenstand und Ausgangslage}

Als die Gartner Research Group 2011 den Begriff «iBPM» lancierte, ging es ihr darum, einen erweiterten Satz an Funktionalitäten von Business-Process-Management (BPM)-Suiten zu definieren. Die Analysten waren zum Schluss gekommen, dass die zum damaligen Zeitpunkt auf dem Markt verfügbaren BPM-Lösungen die gestiegenen Anforderungen der Unternehmen nicht mehr erfüllen konnten ${ }^{1}$. Sie propagierten eine neue Generation von BPM-Suiten, die neben den klassischen Funktionalitäten auch über analytische, soziale und mobile Fähigkeiten verfügen sollten (Gartner 2011).

Doch der Begriff «Prozessintelligenz» war 2011 nicht neu. In der Wirtschafts- und Informatikforschung werden seit Beginn der frühen 2000er-Jahre Definitionen, Konzepte und Referenzarchitekturen für «Business Process Intelligence» (Linden et al. 2010) oder «Operational Intelligence» (Bauer und Schmid 2009) entworfen und bis heute weiterentwickelt.

Vergleicht man die verschiedenen Definitionen, wird der rote Faden deutlich (• Abb. 1.1). Im Wesentlichen geht es darum, die beiden Konzepte Business Process Management und Business Intelligence ${ }^{2} \mathrm{zu}$ vereinen, um deren Methoden und Werkzeuge konzertiert einsetzen zu können. Offensichtlich haben sich die beiden Disziplinen seit den frühen 1990er-Jahren weitestgehend unabhängig voneinander entwickelt, um die wachsende Masse an geschäftsrelevanten Daten auf der einen Seite (BI) und die zunehmend komplexen Wertschöpfungsketten auf der anderen Seite (BPM) beherrschen zu können (- Abb. 1.2). Diese «Paralleluniversen» existieren bis heute in der Praxis: Nicht nur in grossen Organisationen findet BPM und BI entkoppelt und in der Regel in verschiedenen Organisationseinheiten statt. Schnittmengen gibt es vereinzelt beim Reporting oder wenn es darum geht, Prozesskennzahlen in das organisationsweite Performance Management zu integrieren. Innovationen wie Process Mining ${ }^{3}$, das Data Mining und Prozessanalyse kombiniert, geben Hinweise auf das Potenzial, das in einer Kombination beider Disziplinen steckt, vorausgesetzt, man berücksichtigt die speziellen Anforderungen an die Aufbereitung, Analyse und Interpretation operativer Prozessdaten.

\footnotetext{
${ }^{1}$ Lawton (2015) zitiert einen Tweet des ehemaligen Gartner Research VP Jim Sinur, der den Begriff iBPM 2011 prägte: «It was clear that the kind of processes companies wanted to attempt went way beyond the kind of process technologies [that were] available [around] 2011.».

${ }^{2}$ Als Business Intelligence wird die entscheidungsorientierte Sammlung, Aufbereitung und Darstellung geschäftsrelevanter Informationen bezeichnet (Schrödl 2006).

${ }^{3}$ Process Mining ist definiert im Kontext der Fallstudie der Stadt Lausanne in Abschn. 3.3.
} 
) Data-Warehouse (DWH)- bzw. Business-Intelligence (BI)-Werkzeuge verarbeiten zwar (sehr viele) Daten, die in den Prozessen entstehen, haben jedoch in aller Regel keine explizite Vorstellung eines Prozesses, von prozessrelevanten Artefakten oder sonstigen Elementen des BPM-Zyklus (BPM CBOK - Common Body of Knowledge, EABPM 2014, S. 365).

Während BI einen mächtigen Methoden- und Werkzeugkasten für die Sammlung, Aufbereitung und Darstellung von Daten bereitstellt, sind es vor allem die operativen Daten selbst, die in den Prozessen entstehen und die es nutzbar zu machen gilt. Der Fokus in den existierenden Definitionen zu Prozessintelligenz liegt klar auf der Verfügbarmachung und Analyse von operativen Prozessdaten und deren Nutzung zum einen für die Überwachung und Optimierung von Prozessen und zum anderen für die Regelung und Flexibilisierung von Prozessen (Genrich 2008; Hall 2004; Harmon 2004; Hosny 2009; Rowe 2007; Sohail und Dhanapal 2012). \& Schmid, 2009)

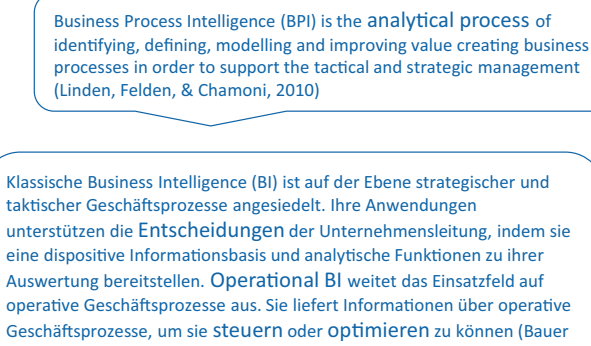
taktischer Geschäftsprozesse angesiedelt. Ihre Anwendungen

unterstützen die Entscheidungen der Unternehmensleitung, indem sie eine dispositive Informationsbasis und analytische Funktionen zu ihrer Auswertung bereitstellen. Operational BI weitet das Einsatzfeld auf operative Geschäftsprozesse aus. Sie liefert Informationen über operative Geschäftsprozesse, um sie steuern oder optimieren zu können (Bauer

BPI refers to the application of various measurement and analysis techniques in the area of business process management. The goal of $\mathrm{BPI}$ is to provide a better understanding and a more appropriate support of a company's processes at design time and the way they are handled at runtime (Hosny, 2009).
The business process intelligence derived from this analysis can then be used to optimize different elements of the predictive enterprise and enable all components to react to changes in the external business environment (Rowe, 2007).

We will use Business Process Intelligence (BPI) to refer to the products being offered by the $\mathrm{BI}$ and Data Warehouse and Packaged Application vendors who seek to drive executive dashboards with data from processes (Harmon, 2004).

Intelligent business process management suites (iBPMSs) enable leading organizations to make their business operations more intelligent by integrating analytics and social and mobile technologies into orchestrated processes. (Gartner, 2011)

- Abb. 1.1 Definitionen für Prozessintelligenz

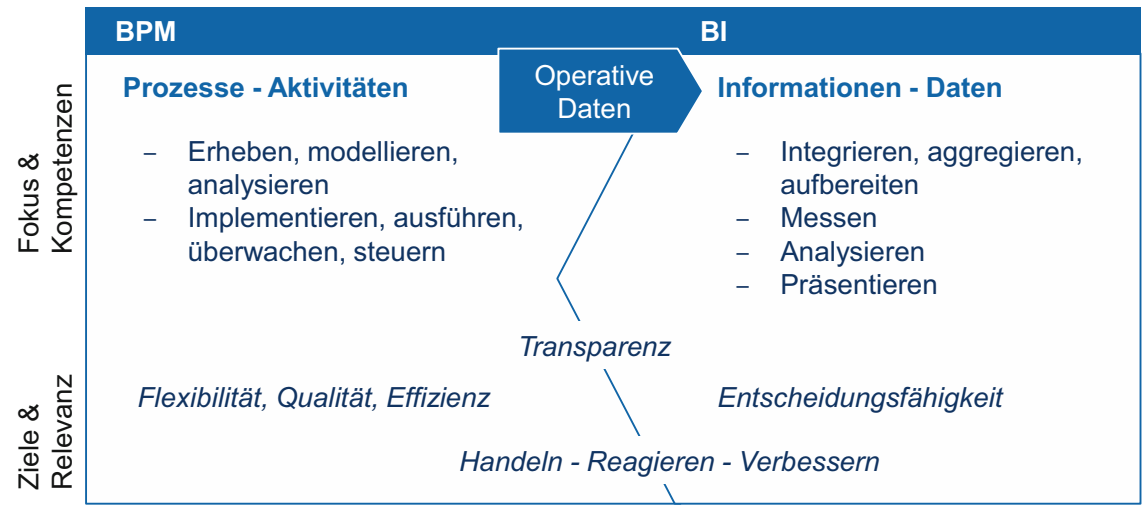

- Abb. 1.2 Business Process Management und Business Intelligence 
In diesem Sinne propagiert Prozessintelligenz eine datenzentrische ${ }^{4}$ Sicht auf das Prozessmanagement und erweitert die klassische Sicht des Prozessmanagements, die sich auf Aktivitäten und Rollen konzentriert. Erkenntnisse aus den Analysen der operativen Daten können sowohl in das Prozessdesign als auch in die Prozessausführung einfliessen.

॥ Process intelligence deals with intelligently using the data generated from the execution of the process (Fundamentals of Business Process Management, Dumas et al. 2013).

Prozessintelligenz liefert somit Lösungsansätze für Unternehmen, die ihr Prozessmanagement in Richtung Operationalisierung und kontinuierliche Optimierung weiterentwickeln wollen. Die ZHAW-Studie «Prozessmanagement 2014» (Brucker-Kley et al. 2014) hat gezeigt, dass Unternehmen im deutschsprachigen Raum ihr Prozessmanagement zunehmend strategisch ausrichten und organisatorisch verankern. Auch IT-Werkzeuge werden verstärkt sowohl für die Prozessmodellierung als auch für die Prozessautomatisierung eingesetzt. Unternehmen scheinen eine klare Vorstellung zu haben, wie sie die Entwicklungsstufen «Transparenz» und «Operationalisierung» erreichen können (- Abb. 1.3). Schwach ausgeprägt hingegen waren das Monitoring der Prozessausführung und die Leistungsmessung, obwohl Effizienzsteigerung nach Standardisierung als wichtigstes Ziel des Prozessmanagements genannt wurde. Entweder sieht das Prozessmanagement in Unternehmen diese Aufgaben nicht als Teil des Leistungsauftrags oder es fehlen Voraussetzungen, um die Prozesse effektiv überwachen und die Erkenntnisse für die kontinuierliche Optimierung nutzen zu können.

\section{GESTALTUNGSEBENEN UND ENTWICKLUNGSSTUFEN EINES GANZHEITLICHEN BPM}

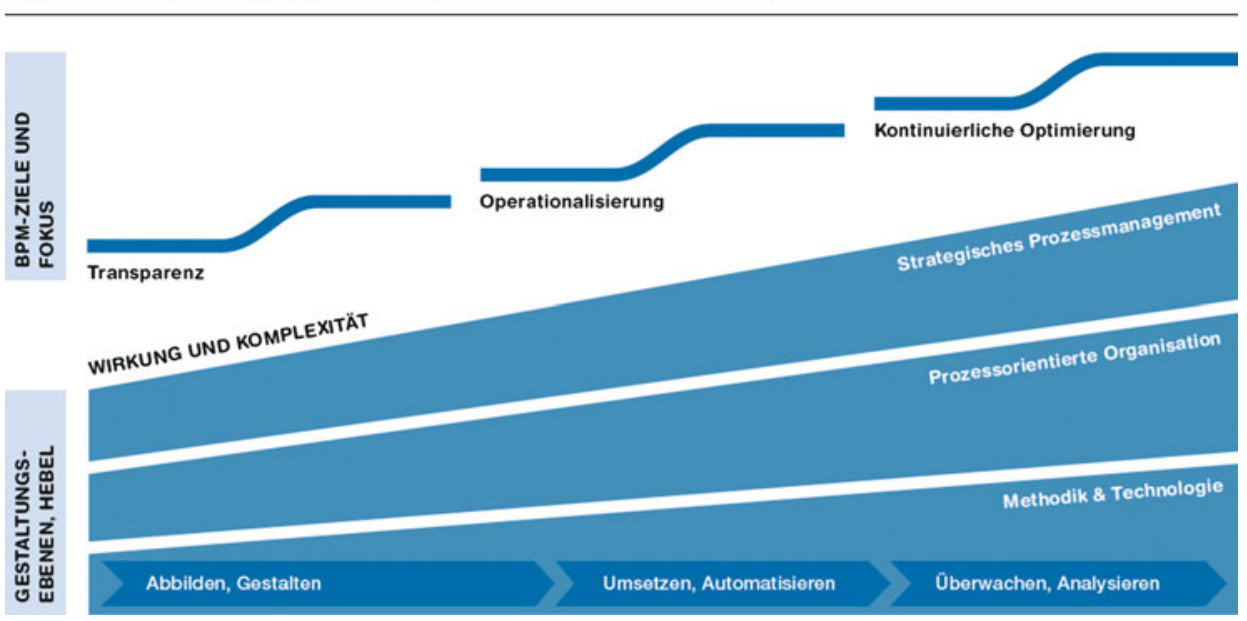

- Abb.1.3 Gestaltungsebenen und Entwicklungsstufen eines ganzheitlichen BPM. (Brucker-Kley et al. 2014)

\footnotetext{
${ }^{4}$ Datenzentrische Ansätze für das Prozessmanagement (= «data-centric, information-centric artifact-centric») finden in der Forschung aktuell verstärkt Beachtung, da sie grosses Potenzial für die Flexibilisierung und Entscheidungsunterstützung in Prozessen versprechen (Brucker-Kley et al. 2014).
} 
An diesem Punkt setzt die BPM-Studie 2015 an. Sie betrachtet «Prozessintelligenz» als einen interessanten Ansatzpunkt, um die Wirksamkeit des Prozessmanagements in Unternehmen zu erhöhen. Zu untersuchen sind:

1. Die Voraussetzungen und der Status quo in der Praxis durch eine quantitative Erhebung: In welcher Ausprägung werden die Konzepte, Methoden und Technologien für Prozessintelligenz, die in der Forschung und von Lösungsanbietern propagiert werden, in Unternehmen im deutschsprachigen Raum bereits eingesetzt?

2. Die Erfolgsmuster in der Praxis durch eine qualitative Erhebung:

Welche Erfahrungen, Lösungsansätze und Resultate können Unternehmen, die bereits Prozessintelligenz-Szenarien umgesetzt haben, aufweisen?

\subsection{Studiendesign und Ergebnisse}

Die Business-Processmanagement-Studie 2015 widmet sich dem Thema «Prozessintelligenz». Ziel der Studie ist es, zum einen Best Practices im Sinne von Erfolgsmustern am Beispiel konkreter Fallbeispiele zu identifizieren und zum anderen den Status quo «Prozessintelligenz» in Unternehmen im deutschsprachigen Raum zu erheben. Welche Methoden und Werkzeuge aus den Fachbereichen BPM und BI werden kombiniert? Was sind die strategischen und operativen Zielsetzungen? Wie stellen Unternehmen sicher, dass Erkenntnisse aus operativen Daten für die Steuerung und Optimierung ihrer Geschäftsprozesse genutzt werden?

\subsubsection{Hypothesen}

Ausgehend von den Erkenntnissen zum Stand von Forschung und Erfahrungswerten aus der Praxis wurden folgende Ausgangshypothesen formuliert:

- Der Lösungsraum für Prozessintelligenz kombiniert Methoden und Werkzeuge für Design, Ausführung, Analyse und Optimierung von Geschäftsprozessen (BPM) mit Methoden und Werkzeugen für die Sammlung, Analyse und Darstellung geschäftsrelevanter Daten (BI), um im Hinblick auf die Unternehmensziele bessere strategische und operative Entscheidungen und eine effizientere, effektivere und flexiblere Prozessausführung zu ermöglichen.

- Der kombinierte Einsatz von Methoden und Werkzeugen aus der BPM- und der BIWelt birgt grosses Nutzenpotenzial. Analytische Fähigkeiten und operative Daten liefern wichtige Erkenntnisse für die Prozesssteuerung und -optimierung. Das Prozessmanagement kann sicherstellen, dass Informationen aus dem unternehmerischen Planungs- und Steuerungszyklus (Ziele, Kennzahlen, Ist-Ermittlung, Ist/Soll-Analyse, Massnahmen) in das Prozessdesign einfliessen und in der operativen Prozessausführung und -steuerung effektiv eingesetzt werden können (strategische Ausrichtung).

- Es existiert bereits ein breites Spektrum von unterschiedlich reifen und unterschiedlich komplexen Methoden und Techniken im BPM- und BI-Lösungsmarkt, um Prozessintelligenz-Anforderungen umzusetzen, das heisst, um die Prozessausführung zu analysieren und die Entscheidungs- und Reaktionsfähigkeit in Prozessen zu erhöhen.

- Unternehmen nutzen die Potentiale eines kombinierten Einsatzes von BPM- und BIMethoden und Technologien vielfach noch nicht. BI und BPM-Kompetenzen sind in vielen Organisationen entkoppelt. 
- Die Verfügbarkeit operativer Daten spielt eine zentrale Rolle in der Umsetzung eines intelligenten Prozessmanagements. Sie können auf der Basis historischer Daten oder in Echtzeit nicht nur aus den Logfiles einer Prozess-/Workflow-Engine, sondern aus verschiedenen Quellen wie Geschäftsapplikationen, Prozess-Engines oder externen Datenquellen bezogen werden. Die Existenz einer unternehmensweiten Integrationsinfrastruktur (EAI, Enterprise Service Bus) unterstützt die Verfügbarkeit aktueller operativer Daten bis hin zu Echtzeitdaten insbesondere für Szenarien, in denen die Prozessausführung flexibilisiert und die Reaktionsgeschwindigkeit auf Prozessereignisse verkürzt werden soll.

- Die technische Basis für die Integration, Aggregation und Analyse grosser Datenmengen sowie zur Aufbereitung der Analyseergebnisse ist insbesondere in kleineren und mittleren Unternehmen nur vereinzelt oder in geringer Ausprägung vorhanden.

- Es gibt Unternehmen, die bereits Prozesse und Prozessdaten (historisch und real-time) analysieren und auf dieser Grundlage Prozesse optimieren und die Prozessausführung steuern. Dies sind primär Grossunternehmen, die hierfür komplexe und mächtige Technologien einsetzen.

- Diese Lösungen bauen überwiegend auf Geschäftsapplikationen oder Core Business Engines (z. B. ERP-Systemen oder Branchenlösungen wie Kernbankensystemen) und komplexen Datenaggregations- und Integrationslösungen auf. Ein integrierter Einsatz von BPM-Systemen (konkret Process-Engines, Workflowsystems) und BI-Lösungselementen (z. B. Datawarehouses, Datamining, Predictive Analytics, Dashboards) ist noch wenig ausgeprägt. Standard-Schnittstellen (z. B. für Big und Open Data Integration) sind kaum vorhanden.

- Die relevanten Anwendungsszenarien für Prozessintelligenz hängen von den strategischen und operativen Prämissen und Zielsetzungen eines Unternehmens oder eines konkreten Geschäftsvorhabens ab. Unternehmen können die Nutzenpotenziale von Prozessintelligenz ausschöpfen, um

- Prozessleistung zu überwachen und zu optimieren;

- Prozessrisiken zu minimieren und die Konformität von Prozessen zu prüfen (Soll/IstExecution-Gaps, Compliance, Qualitätsmanagement);

- Kunden-/Kontextorientierung zu verbessern;

- Entscheide in Prozessen zu unterstützen;

- Prozessstrukturen zu erkennen und zu rekonstruieren (Transparenz);

- Prozesse agiler zu machen (Flexibilität);

- Potenziale für neue Produkte, Dienstleistungen oder Geschäftsmodelle zu erkennen (Innovation).

\subsubsection{Vorgehen}

Um die Hypothesen zu prüfen, wurde eine Kombination aus qualitativer und quantitativer Forschung gewählt. - Abb. 1.4 skizziert das Vorgehen. - Tab. 1.1 fasst das Studiendesign mit den drei wesentlichen Studienelementen, Forschungsfragen und Ergebnissen zusammen. 


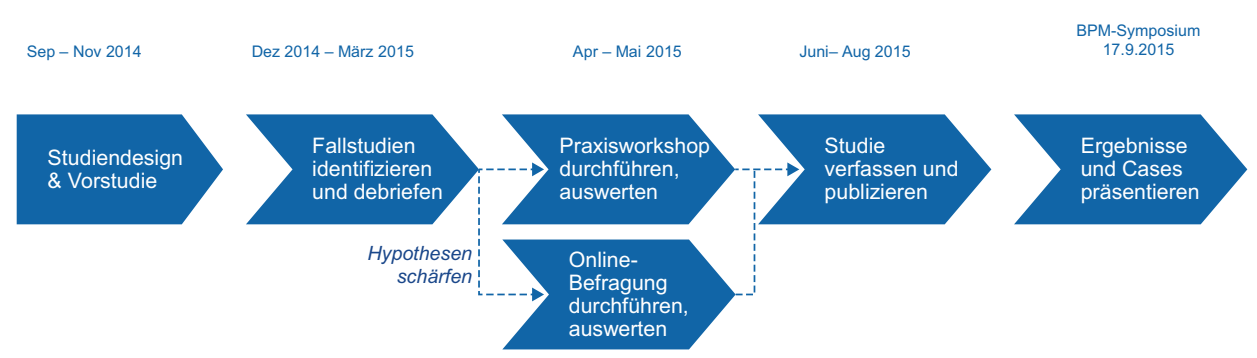

- Abb. 1.4 Vorgehen zur Konzeption und Durchführung der Studie

- Tab. 1.1 Forschungsfragen, Vorgehen und Ergebnisse

\begin{tabular}{|c|c|c|}
\hline Forschungsfragen & Vorgehen & Ergebnisse (Studie) \\
\hline Was ist Prozessintelligenz? & $\begin{array}{l}\text { Vorstudie: } \\
\text { Stand der Forschung «Prozessintelligenz» } \\
\text { Analyse von Intelligenzdefinitionen aus der } \\
\text { Humanpsychologie und der Künstlichen Intelli- } \\
\text { genz; } \\
\text { - Auswahl des Konzepts «Erfolgsintelligenz» } \\
\text { nach Sternberg }\end{array}$ & $\begin{array}{l}\text { Rahmenwerk «Prozessin- } \\
\text { telligenz» ( } \text { Abschn. 1.3) }\end{array}$ \\
\hline $\begin{array}{l}\text { Welche Lösungsansätze, } \\
\text { Erfahrungen und Erfolgs- } \\
\text { muster gibt es bereits in } \\
\text { Unternehmen? }\end{array}$ & $\begin{array}{l}\text { Qualitativ: } \\
\text { Debriefings mit Fallstudienkandidaten } \\
\text { - Ganztägiger Praxis-Workshop mit } 5 \text { Anwen- } \\
\text { derunternehmen, um Early/Best Practices } \\
\text { «Prozessintelligenz» zu identifizieren }\end{array}$ & $\begin{array}{l}\text { Fallstudien «Prozessintelli- } \\
\text { genz» ( Kap. 3-7) } \\
\text { Fazit: Erfolgsmuster } \\
(\triangleright \text { Abschn. 9.2) }\end{array}$ \\
\hline $\begin{array}{l}\text { Wie ausgeprägt ist der } \\
\text { Einsatz von Methoden } \\
\text { und Technologien in Un- } \\
\text { ternehmen? Vor welchem } \\
\text { Hintergrund? }\end{array}$ & $\begin{array}{l}\text { Quantitativ: } \\
\text { Online-Befragung (April-Mai 2015) }\end{array}$ & $\begin{array}{l}\text { Umfrage-Ergebnisse } \\
\text { ( Kap. 8) } \\
\text { Fazit: Status quo } \\
\text { «Prozessintelligenz» } \\
(\triangleright \text { Abschn. 9.1) }\end{array}$ \\
\hline
\end{tabular}

\subsection{Das Rahmenwerk: Prozessintelligenz $=$ Erfolgsintelligenz}

Wissenschaft und Lösungsanbieter sehen Prozessintelligenz als ein Konzept, das durch eine Kombination von Methoden, Technologien und operativen Prozessdaten realisiert wird. Doch ist das alles? Ist Prozessintelligenz nichts anderes als Datensammlung und Analytik? Oder braucht es noch andere Fähigkeiten und Rahmenbedingungen, um Geschäftsprozesse wirksamer gestalten und ausrichten zu können?

Die vorliegende Studie geht von der Hypothese aus, dass es mehr braucht als Datensammlung und Analytik, um die Nutzenpotenziale von Prozessintelligenz ausschöpfen zu können. In diesem Kapitel wird ein erweitertes Rahmenwerk für Prozessintelligenz vorgeschlagen, das auf dem triarchischen Intelligenz-Modell des Psychologen Robert J. Sternberg basiert, der den 
Begriff der «Erfolgsintelligenz» prägte ${ }^{5}$. Dieses Rahmenwerk dient als Grundlage, um

- relevante Anwendungsszenarien und Kandidaten für den Praxisworkshop zu identifizieren;

- den Lösungsraum für Prozessintelligenz und dessen Einflussfaktoren, Methoden und Werkzeuge zu strukturieren;

- die Ergebnisse der Online-Befragung auszuwerten.

\subsubsection{Erfolgsintelligenz}

Was ist Intelligenz? Können Organisationen, Prozesse oder IT-Lösungen intelligent sein? Unter Intelligenz wird in erster Linie die kognitive Leistungsfähigkeit eines Menschen verstanden. Doch auch in der Humanpsychologie gibt es unterschiedliche Ansichten, was Intelligenz ist und wovon sie beeinflusst wird (Plucker 2014).

Robert J. Sternberg $\left({ }^{*} 1949\right)$ zählt zu den renommiertesten Psychologen des 20. Jahrhunderts und das, obwohl ihm seine Lehrer zunächst keine gute Prognose erteilten. Sternberg war bereits früh in seiner Schulkarriere, bedingt durch Prüfungsangst, Opfer von Intelligenztests geworden, die ihm einen geringen IQ bescheinigten. Eine erfahrene Lehrerin erkannte aber das Potenzial des Jungen und stellte seine Bildungskarriere sicher, die ihn an renommierte Universitäten wie Yale und Stanford und bis zum Professorentitel führte. Von seinen Erlebnissen geprägt, studierte Sternberg Psychologie und spezialisierte sich auf Entwicklungspsychologie, Psychometrie und Intelligenzforschung. Sternberg gehörte zu einer neuen Generation von Entwicklungspsychologen, die seit Beginn der 1980er-Jahre den traditionellen Intelligenzbegriff anzweifelten, der davon ausging, dass Intelligenz eine feststehende, kaum beeinflussbare und über den IQ messbare Eigenschaft des Menschen sei. Vielmehr sahen sie Intelligenz als ein Set an Fähigkeiten (multiple intelligences ${ }^{6}$ ), die sich permanent entwickeln. Sternberg sah, neben der analytischen Intelligenz insbesondere die Relevanz der praktischen Intelligenz, die sich darin zeigt, wie gut ein Mensch mit seinen Rahmenbedingungen zurechtkommt. Komplettiert wird sein triarchisches Modell durch die kreative Intelligenz, die einen Menschen zum Lernen motiviert und in die Lage versetzt, Neues zu schaffen, aber auch Unwichtiges von Wichtigem zu unterscheiden und Schwächen durch Stärken zu kompensieren (Sternberg 1985). Später fasste Sternberg die drei Fähigkeiten seines Modells unter dem Begriff «Erfolgsintelligenz» zusammen, dessen Essenz er 2014 in einem Videointerview formulierte:

I) Successful intelligence is your ability to figure out what you want to do with your life and to succeed in doing it given the constraints of the environment in which you live (Sternberg 2014).

Entscheidend für Erfolgsintelligenz sind demnach nicht isolierte Fähigkeiten oder analytische Intelligenz, wie sie in klassischen Schulnoten und IQ-Tests gemessen wird, sondern die Erreichung von Zielen, die für eine Person oder einen bestimmten Kontext wichtig sind. - Abb. 1.5 skizziert die drei Elemente des Modells, kreative, analytische und praktische Intelligenz. In der Weiterentwicklung seines Modells spricht Sternberg nicht mehr von Intelligenzen,

\footnotetext{
${ }^{5}$ Erstmalig publiziert in Sternberg, Toward a triachic theory of human intelligence (1984), weiterentwickelt (1988) unter dem Begriff Erfolgsintelligenz publiziert in: Sternberg, Successful Intelligence: how practical and creative intelligence determine success in life (1997), ins Deutsche übersetzt: Sternberg, Erfolgsintelligenz. Warum wir mehr brauchen als EQ und IQ (1998).

${ }^{6}$ Wesentlich geprägt von Howard Gardner (Gardner 1983).
} 


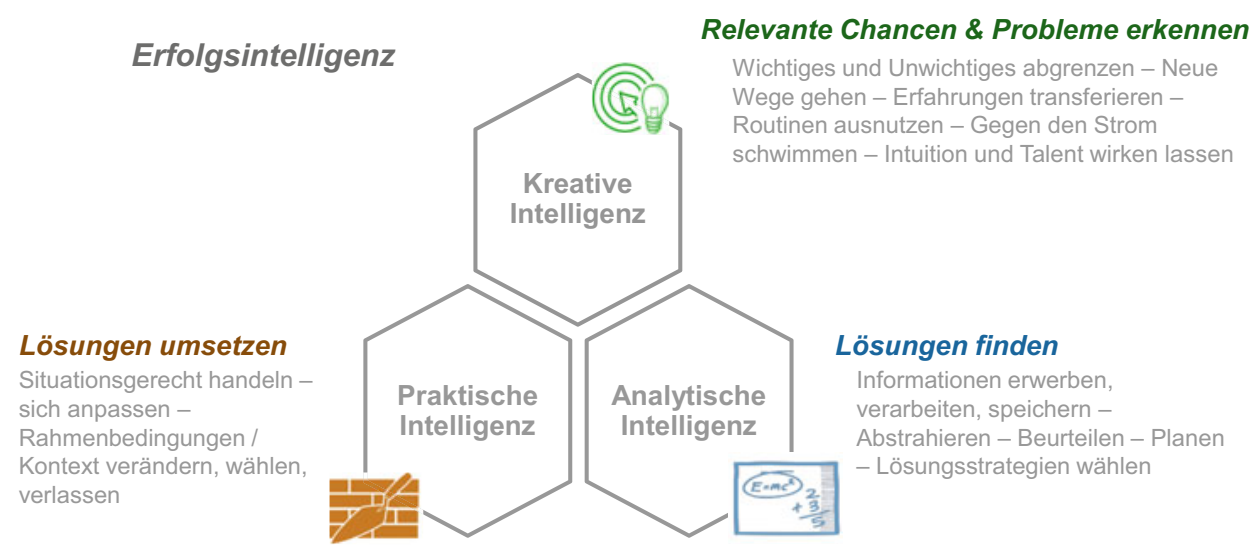

- Abb. 1.5 Erfolgsintelligenz nach Robert J. Sternberg. (eigene Darstellung)

sondern von Fähigkeiten, da aus seiner Sicht keine dieser Fähigkeiten isoliert intelligent oder erfolgreich macht (Sternberg 1997).

\subsubsection{Prozessintelligenz $=$ Erfolgsintelligenz für Organisationen}

Was macht Sternbergs Modell von Erfolgsintelligenz relevant und anwendbar für die vorliegende Studie? Auch Prozessintelligenz muss zielgerichtet eingesetzt werden und verlangt eine integrierte Betrachtung verschiedener Fähigkeiten, die Organisationen nicht per se besitzen sondern kontinuierlich und in einem gegebenen, aber gestaltbaren Kontext entwickeln müssen.

Folgende Eigenschaften des Erfolgsintelligenzmodells machen seine Anwendung auf einen organisatorischen Kontext und konkret das Geschäftsprozessmanagement wertvoll:

\section{- Lernkurve}

Nach Sternberg ist Intelligenz keine feste Grösse, sondern ein Set an Fähigkeiten, das sich kontinuierlich entwickelt. Diese Sicht entspricht dem Lebenszyklus eines kontinuierlichen Prozessmanagements, das auf kontinuierliche Verbesserung ausgerichtet ist.

\section{- Erfahrung und Wissensmanagement}

Für jede der drei Intelligenzen beziehungsweise Fähigkeiten definiert Sternberg Komponenten im Sinne kognitiver Prozesse, die die jeweilige Intelligenz ermöglichen. So hängt analytische Intelligenz nicht nur von der sogenannten "performance component» ab, die die Lösungsstrategien bereitstellt, sondern auch von der «knowledge acquisition component», die dem Wissenserwerb dient. Sie hängt eng zusammen mit der «transfer component» der kreativen Intelligenz, die sicherstellt, dass das erworbene Wissen auf neue Situationen angewandt werden kann. Dies entspricht der Zielsetzung von Prozessintelligenz, Erfahrungswissen aus vergangenen Prozessinstanzen für die Entscheide und Reaktionen in zukünftigen Prozessinstanzen nutzbar zu machen. Finden solche Transfers systematisch statt, unterstützt Prozessintelligenz die lernende Organisation.

\section{- Informationsverarbeitung}

Sternberg ist mit seinem triarchischen Modell einer der wichtigsten Vertreter des Informationsverarbeitungsansatzes in der Intelligenztheorie. Die «information processing 


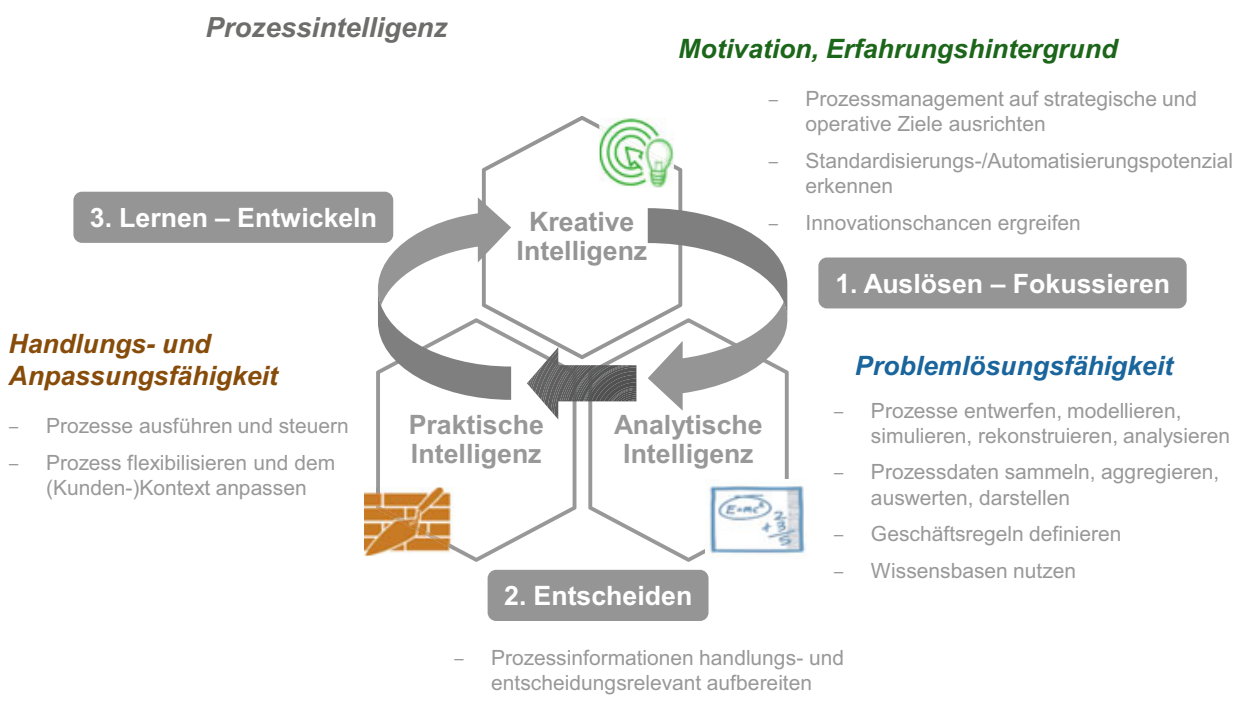

- Abb.1.6 Anwendung des triarchischen Modells «Erfolgsintelligenz» auf das Prozessmanagement

theory» verwirft die Idee von grundlegenden Faktoren der Intelligenz. Sie befasst sich vielmehr mit den während der Informationsverarbeitung ablaufenden kognitiven Prozessen (welche Art, wie akkurat, mit welcher mentalen Repräsentation). Da Prozessintelligenz in hohem Masse von Modellen, Systemen und der Verarbeitung von Prozessdaten abhängt, erscheint diese Ausrichtung hilfreich.

- Kontext

Ein zentrales Element in Sternbergs Modell ist die Anerkennung des Kontextes, der selbstverständlich einen Einfluss auf die Möglichkeiten, Erwartungen und Ziele eines Menschen und seines Umfeldes haben. Der Kontext gilt aber als gestaltbar, das Modell stellt mit den drei Komponenten «Change/Adapt/Select» explizit Optionen zur Verfügung, einen Kontext zu verändern, sich dem Kontext anzupassen oder einen Kontext gezielt zu wählen beziehungsweise zu verlassen. Der Kontext spielt auch für das Prozessmanagement in mehrerer Hinsicht eine wesentliche Rolle. Er bestimmt die Zielsetzungen für das Prozessmanagement und legt fest, worauf sich das Prozessmanagement oder einzelne Initiativen fokussieren. Der Kontext von Geschäftsprozessen im Sinne von Rahmenbedingung (z. B. Ressourcen, Aufbauorganisation, IT-Unterstützung) muss gestaltet werden. In Bezug auf konkrete Prozessinstanzen stellt der Kontext eine wesentliche Information dar, wenn es darum geht, Prozessverläufe situationsgerecht anpassen zu können.

- Abb. 1.6 fasst die Anwendung des Erfolgsintelligenzmodells auf das Prozessmanagement zusammen und skizziert die Fähigkeiten, die Prozessintelligenz in den drei Bereichen kreative, analytische und praktische Intelligenz bereitstellen kann.

Ausgehend von dieser erweiterten Sicht auf das Thema Prozessintelligenz gilt die folgende Definition von Prozessintelligenz als Arbeitsgrundlage für die vorliegende Studie:

I) Prozessintelligenz ist mehr als Datensammlung und Analytik. Prozessintelligenz umfasst die kreativen, analytischen und praktischen Fähigkeiten, mit denen eine Organisation ihre Geschäftsprozesse gestaltet, ausführt, überwacht und fortlaufend weiterentwickelt. 


\section{Literatur}

Bauer, A., \& Schmid, T. (2009). Was macht Operational Bl aus? BI-Spektrum, 4(1), 13-14.

Brucker-Kley, E., Kykalova, D., Pedron, C., Luternauer, T., \& Keller, T. (2014). Business Process Management 2014: Status-quo und Perspektiven eines ganzheitlichen Geschäftsprozessmanagement. Zürich: vdf Hochschulverlag.

Dumas, M., La Rosa, M., Mendling, J., \& Reijers, H. A. (2013). Fundamentals of business process management. Heidelberg: Springer.

EABPM (2014). Business process management common body of knowledge - BPM CBOK version 3.0. Giessen: Dr. Götz Schmidt.

Gardner, H. (1983). Frames of mind: the theory of multiple intelligence. New York: Basic Books.

Gartner (2011). BPM suites evolve into intelligent BPM suites. Stamford: Gartner Research.

Genrich, M. K. (2008). Challenges for business process intelligence: discussions at the BPI workshop 2007. In A. ter Hofstede \& B. P.-Y. Benatallah (Hrsg.), BPM workshops 2007. LNCS, (Bd. 4928, S. 5-10). Heidelberg: Springer.

Hall, C. (2004). Business process intelligence. Business Process Trends, 2(6), 1-11.

Harmon, P. (2004). Business performance management: the other BPM. Business Process Trends, 2(7), 1-12.

Hosny, H. (2009). Business process intelligence. In Dimensions of business process intelligence. ATIT 2009, Cairo. (S. 213).

Lawton, G. (2015). Ten top iBPM use cases. Von Techtarget. http://searchsoa.techtarget.com/photostory/ 2240239333/Ten-top-iBPM-use-cases/1/Intelligent-business-process-management-emerges. Zugegriffen: 22.07.2015

Linden, M., Felden, C., \& Chamoni, P. (2010). Dimensions of business process intelligence. In M. zur Muehlen \& J. Su (Hrsg.), Business process management workshops, BPM 2010 international workshops and education track. Hoboken, 09.2010. LNBIP 66. (S. 208-2013). Heidelberg: Springer.

Plucker, J. A. (2014). Human intelligence: Historical influences, current controversies, teaching resources. http:// www.intelltheory.com. Zugegriffen: 22.07.2015

Rowe, A. (2007). From business process management to business process intelligence. DM Review, 46.

Schrödl, H. (2006). Business intelligence. München, Wien: Hanser.

Sohail, A., \& Dhanapal, P. D. (2012). A gap between business process intelligence and process redesign. 2012 International Conference on Computer \& Information Science. (S. 136-142).

Sternberg, R. J. (1984). Toward a triachic theory of human intelligence. Bahavioral and Brain Sciences, 7.

Sternberg, R. J. (1985). Beyond IQ: a triarchic theory of intelligence. Cambridge: Cambridge University Press.

Sternberg, R. J. (1988). The triarchic mind: a new theory of intelligence. New York: Viking Press.

Sternberg, R. J. (1997). Successful intelligence: how practical and creative intelligence determine success in life. New York: Penguin, Putnam.

Sternberg, R. J. (1998). Erfolgsintelligenz. Warum wir mehr brauchen als EQ und IQ. München: Lichtenberg.

Sternberg, R. J. (2014). Successful Intelligence. Video. https://youtu.be/ow05B4bjGWQ. Zugegriffen: 04.06.2015

Open Access Dieses Kapitel wird unter der Creative Commons Namensnennung 4.0 International Lizenz (http://creativecommons.org/licenses/by/4.0/deed.de) veröffentlicht, welche die Nutzung, Vervielfältigung, Bearbeitung, Verbreitung und Wiedergabe in jeglichem Medium und Format erlaubt, sofern Sie den/die ursprünglichen Autor(en) und die Quelle ordnungsgemäß nennen, einen Link zur Creative Commons Lizenz beifügen und angeben, ob Änderungen vorgenommen wurden.

Die in diesem Kapitel enthaltenen Bilder und sonstiges Drittmaterial unterliegen ebenfalls der genannten Creative Commons Lizenz, sofern sich aus der Abbildungslegende nichts anderes ergibt. Sofern das betreffende Material nicht unter der genannten Creative Commons Lizenz steht und die betreffende Handlung nicht nach gesetzlichen Vorschriften erlaubt ist, ist für die oben aufgeführten Weiterverwendungen des Materials die Einwilligung des jeweiligen Rechteinhabers einzuholen.

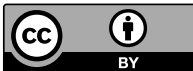

